\title{
Correction: Diabetic retinopathy and diabetic macular oedema pathways and management: UK Consensus Working Group
}

\author{
Winfried M. Amoaku - Faruque Ghanchi - Clare Bailey · Sanjiv Banerjee - Somnath Banerjee - Louise Downey • \\ Richard Gale - Robin Hamilton - Kamlesh Khunti - Esther Posner • Fahd Quhill • Stephen Robinson - Roopa Setty • \\ Dawn Sim $\cdot$ Deepali Varma $\cdot$ Hemal Mehta
}

Published online: 22 July 2020

(c) The Author(s), under exclusive licence to The Royal College of Ophthalmologists 2020

Correction to: Eye

https://doi.org/10.1038/s41433-020-0961-6

Since the online publication of the above article, the authors have noted that the in Section 1: Scope, the text: "NICE does not recommend licensed pharmacological therapies for DMO in eyes with CRT $<400 \mu \mathrm{m}$, as such treatments although clinically effective, are not considered cost-effective (NICE TA274; TA346; TA349) [14-16]. The SMC uses VA criteria rather than OCT parameters [17-19]. In particular, dexamethasone implant (Ozurdex, Allergan) is recommended only in eyes with DMO that are pseudophakic, and unresponsive to other therapies." should read: "NICE does not recommend licensed anti-VEGF therapies for DMO in eyes with CRT $<400 \mu \mathrm{m}$, as such treatments, although clinically effective, are not considered cost-effective (NICE TA274; TA346) [14-16]. The SMC uses VA criteria rather than OCT parameters [17-19]. In particular, dexamethasone implant (Ozurdex, Allergan) is recommended by NICE only in eyes with DMO that are pseudophakic, and unresponsive or unsuitable for noncorticosteroid therapies (NICE TA349), whereas the SMC recommends treatment with Ozurdex in eyes that are not sufficiently responsive to other therapies irrespective of lens status, whilst recommendation for fluocinolone is similar to that from NICE."

In Figure 1, the box "History of $\mathrm{CNV}$ or Injection Burden" and the legend text that reads " $\mathrm{CNV}=$ choroidal neovascular membrane" should read "History of CVE or Injection Burden" and "CVE = cardiovascular events", respectively.

In Section 12: The management of DMO, the text: "Dexamethasone implant (Ozurdex). NICE TA349 [15] recommended it as an option for treating chronic DMO that is insufficiently responsive to available therapies in pseudophakic eyes, based on the results from the MEAD study [320]." should read: "Dexamethasone implant (Ozurdex).
NICE TA349 [15] recommended it as an option for treating DMO that is insufficiently responsive to (or unsuitable for) available therapies in pseudophakic eyes, based on the results from the MEAD study[320]".

In the next section, SMC guidance (applicable to Scotland only), the text: "For the dexamethasone and fluocinolone implants, these are approved for pseudophakic eyes where there has been insufficient response (or not suitable) for non-corticosteroid therapy $[19,321] . "$ should read: "For the dexamethasone implants, these are approved for pseudophakic eyes or where there has been insufficient response to (or the patients are unsuitable for) noncorticosteroid therapy (i.e. may be permitted for some phakic eyes).[19, 321]. For the fluocinolone implants, these are approved for pseudophakic eyes only, that have been insufficiently responsive to available therapies."

Additionally, the section "Recommendation for eyes with CI-DMO not meeting NICE or SMC criteria", the text: "The consideration for switching to intravitreal steroid treatment for insufficiently responsive eyes is discussed above (Fig. 3)." should read, "The consideration for switching to intravitreal steroid treatment for insufficiently responsive eyes is discussed above (Fig. 4)."

In the Recommendations section of Section 13: Response to DMO therapies, the text: "If the eye is NOT pseudophakic, consider phaco/IOL if cataract is present followed by Ozurdex or Iluvien or. (Level 1, B)." should read: "If the eye is NOT pseudophakic, consider phaco/IOL if cataract is present followed by Ozurdex or Iluvien. (Level 1, B)." and the text: "Where the eye is NOT pseudophakic, and there is no significant cataract, and the DMO is chronic or inadequately responsive to anti-VEGFs, or the patient is pregnant or has other contraindications to anti-VEGF therapies (such as a recent cardiovascular event), it is appropriate to consider dexamethasone implant (Ozurdex) injection." should instead read: "Where the eye is NOT pseudophakic, and there is no significant cataract, and the 
DMO is inadequately responsive to anti-VEGF therapy, or the patient is pregnant or is unsuitable to receive anti-VEGF therapies (such as a recent cardiovascular event), it is appropriate to consider dexamethasone implant (Ozurdex) injection. These caveats are not required in Scotland, where the SMC allows the use of Ozurdex irrespective of the lens status."
All instances of "Iluvien" in Fig. 4 should read "Iluvien**" and the text at the bottom of the figure should have the text “***lluvien is only recommended in patients insufficiently responsive to available therapies. Avoid the use of Iluvien during pregnancy." added to it.

The authors apologise for any inconvenience caused by these errors.

Summary of changes:

\begin{tabular}{|c|c|}
\hline Section & Old text \\
\hline Section 1: Scope, the text & 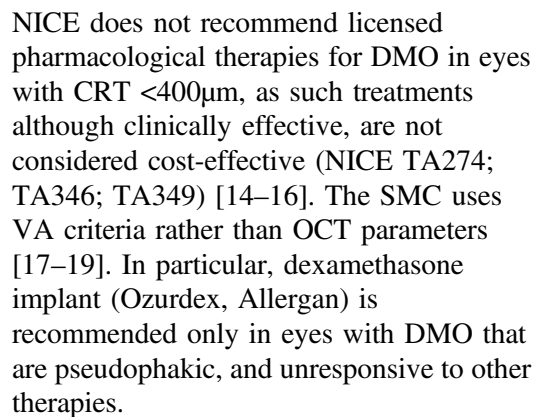 \\
\hline
\end{tabular}

In Figure 1, box

In Section 12: The management of DMO, the text:

In the next section, SMC guidance (applicable to Scotland only), the text:

Recommendation for eyes with CI-DMO not meeting NICE or SMC criteria

Recommendations section of Section 13: Response to DMO therapies
History of CNV or Injection Burden $\mathrm{CNV}=$ choroidal neovascular membrane

Dexamethasone implant (Ozurdex). NICE TA349 [15] recommended it as an option for treating chronic DMO that is insufficiently responsive to available therapies in pseudophakic eyes, based on the results from the MEAD study [320].

For the dexamethasone and fluocinolone implants, these are approved for pseudophakic eyes where there has been insufficient response (or not suitable) for non-corticosteroid therapy [19, 321]."

The consideration for switching to intravitreal steroid treatment for insufficiently responsive eyes is discussed above (Fig. 3)

If the eye is NOT pseudophakic, consider phaco/IOL if cataract is present followed by Ozurdex or Iluvien or. (Level 1, B)

Where the eye is NOT pseudophakic, and there is no significant cataract, and the DMO is chronic or inadequately responsive to anti-VEGFs, or the patient is pregnant or has other contraindications to anti-VEGF therapies (such as a recent cardiovascular event), it is appropriate to consider dexamethasone implant (Ozurdex) injection.

Fig. 4
Iluvien
New text

NICE does not recommend licensed anti-VEGF

therapies for DMO in eyes with CRT $<400 \mu \mathrm{m}$, as such treatments, although clinically effective, are not considered cost-effective (NICE TA274; TA346) [1416]. The SMC uses VA criteria rather than OCT parameters [17-19]. In particular, dexamethasone implant (Ozurdex, Allergan) is recommended by NICE only in eyes with DMO that are pseudophakic, and unresponsive or unsuitable for non-corticosteroid therapies (NICE TA349)", whereas the SMC recommends treatment with Ozurdex in eyes that are not sufficiently responsive to other therapies, irrespective of lens status, whilst recommendation for fluocinolone is similar to that from NICE.

History of CVE or Injection Burden

$\mathrm{CVE}=$ cardiovascular events

Dexamethasone implant (Ozurdex). NICE TA349

[15] recommended it as an option for treating DMO that is insufficiently responsive to (or unsuitable for) available therapies in pseudophakic eyes, based on the results from the MEAD study.[320]

For the dexamethasone implants, these are approved for pseudophakic eyes or where there has been insufficient response to (or the patients are unsuitable for) non-corticosteroid therapy (i.e. may be permitted for some phakic eyes).[19, 321]. For the fluocinolone implants, these are approved for pseudophakic eyes only, that have been insufficiently responsive to available therapies.

The consideration for switching to intravitreal steroid treatment for insufficiently responsive eyes is discussed above (Fig. 4)

If the eye is NOT pseudophakic, consider phaco/IOL if cataract is present followed by Ozurdex or Iluvien. (Level 1, B)

Where the eye is NOT pseudophakic, and there is no significant cataract, and the DMO is inadequately responsive to anti-VEGF therapy, or the patient is pregnant or is unsuitable to receive anti-VEGF therapies (such as a recent cardiovascular event), it is appropriate to consider dexamethasone implant (Ozurdex) injection. These caveats are not required in Scotland, where the SMC allows the use of Ozurdex irrespective of the lens status.

Iluvien**

“***luvien is only recommended in patients insufficiently responsive to available therapies. Avoid the use of Iluvien during pregnancy." added to it. 